\title{
UNIFORMITY IN DOSAGE OF RADIUM EMANATION.
}

WM. JAY SCHIEFFELIN, PH. D.

Radium emanation is assuming importance as a therapeutic agent. The Council on Pharmacy of the American Medical Association, has listed radium and its emanation among New and Non-Official Remedies; an increasing number of physicians are using the emanation in their practice, and articles and advertisements on the subject, are appearing in the medical journals. Since radium and its emanation, are becoming recognized as belonging in the Materia Medica, their production and properties and the standardization of their preparations, come within the scope of Pharmacy.

Radium is prepared from carnotite (vanadate of uranium and potassium), uraninite or pitchblende (uranium oxide), and samarskite (columbate and tantalate of uranium and yttrium). Radium has an atomic weight of 226 , and resembles barium in its chemical properties.

In its characteristic property of radio-activity, radium is sublimely superior to its environment: whether in its natural minerals or isolated from them, and in all of its chemical compounds, it is constantly emitting alpha rays and emanation, at a uniform rate, and there is no known way of influencing or halting this activity, which is not affected by the extremes of heat and cold, by pressure or the strongest re-agents. This radio-activity shows the energy which results from the disintegration or transmutation of radium into elements of lower atomic weights. The energy resulting from the resolution of an element into its constituent parts, is vastly greater than that resulting from chemical combination.

The largest amount of energy released in any known chemical reaction, is from the combination of an equal volume of hydrogen and oxygen to form water, but the emapation of radium, in its successive transformations, accompanied by the expulsion of alpha rays, yields more than ten million times as much energy. Ninety-five percent. of the energy in the radiation from radium and its subsequent products, is in the form of the alpha radiation, a corpuscular radiation in which the corpuscles or alpha particles are positively charged helion ions.

The statement that there are one hundred and thirty-six million alpha particles, emitted by one milligram of radium each second, to one who studied chemistry twenty-five years ago, seems almost humorous, but the description of the apparatus for counting the alpha radiations is so simple that a fourteen year old child can understand it.

Counting the number of particles per second, is possible, because physicists have been able to determine, exactly, the diameter of the sphere, on whose surface the particles are far enough apart to count them.

They place a fraction of a gram of radium in a tube, some thirteen feet long, from which the air is exhausted, which tube has a small orifice at the end, to prevent more than a certain number of alpha particles from coming through, and on the other side of the orifice, is a delicate electrometer with a silevered quartzfibre mirror, by which a point of light is reflected. Each atom of helium. 
charged with positive electricity, causes a tremor in the quartz-fibre, which is magnified by the reflected point of light and recorded on a photograph film. They then have the data required for their calculation,- the weight of the radium, the length of the radius and the area of the orifice through which the little particles went, and as they know this is taking place in every direction; that this amount of radium is shooting out these little particles, (actual, visible, corpuscular particles) in every direction all the time, it is a simple calculation to determine what the number of radiations on the surface of the sphere is. You know radium is magnetic, and they adjust the distance by moving the fragment of radium backward and forward, by use of a magnet outside of the glass tube. By means of this apparatus, Rutherford found that there are one hundred and thirty-six million separate alpha particles radiated from one milligram per second. The spinthariscope renders a confirmation of this calculation possible.

I have here a modified spinthariscope, called the radiosmitoscope, which is adjusted so the zinc sulphide screen can be moved out so you can see the radium particles emanating from other bodies outside. I suppose you know how the spintariscope is made.

A needle point is touched to the inside surface of the tube which has contained radium; enough radium adheres to the point of that needle to radiate sparks for 1800 years. Seen through a low-power microscope they are like sparks from a blacksmith's anvil radiating continuously.

A milligram of radium expels one hundred and thirty-six million separate alpha particles per second, which are made visible in a spinthariscope. The alpha rays emitted from one three thousand-millionth of a grain of radium, can be detected by the gold leaf electroscope. The rays are given out uniformly, in all directions, in the form of continuous volleys of tiny projectiles travelling at a rate of 12,000 miles per second. Their range is nearly three inches in air and many yards in a vacuum. They are but slightly penetrating,-being absorbed by thin sheets of aluminum, paper or glass--but pass through a film of glass exceedingly thin. It has been shown that they are atoms of helium charged with positive electricity.

Now, here is a very important thing from a pharmaceutical point of view. Only a fraction of the rays, are set free from solid radium compounds; only a fraction of them come out. The bulk of them are not set free, but are occluded in the salt of radium, or the mineral in which the radium is contained. In order to obtain the largest yield, you must have the largest possible surface. If you have a crystal of chloride of radium, not nearly as many of these little alpha radiations come out as if you dissolved that crystal and spread it out as widely as possible on a surface. If that is done, the number will be very much increased.

Now, the same thing applies to insoluble salts. If you have sulphate of radium, you will not get the greatest amount of emanation from it unless you powder it to the finest degree and spread it out as far as possible. Otherwise, you may get two percent. only, instead of possibly ten percent. of the total emanation available.

The emanation is a gas which, in turn, steadily disintegrates into alpha parti- 
cles and radium $A$, from which, in the same way, come Radium $B, C, D, E$, and $F$, in succession. It is from these products, especially Radium $C$, that the beta and gamma rays are given off. The beta rays are electrons of negative electricity, the same as the cathode rays, except that the velocity of the beta particles is much greater, approaching the velocity of light, 186,000 miles a second.

The gamma rays are not considered to be particles of matter, but are waves in the ether, similar to the X-rays. They are far more penetrating than the alpha and beta rays, and are used in the external application of radium in cancer, the others being easily excluded by thin metal filters.

The emanation has an atomic weight of 222 and a characteristic bright line spectrum. It belongs in the group of inert monatomic gases with helium and argon. It is not absorbed by any known re-agent and shows no power of chemical combination. The emanation is one hundred thousand times as active, weight for weight, as radium. Like other gases, it can be collected, confined, and handled, in ordinary glass containers. This is usually done only when it is mixed with enormously greater volumes of air or other gases. Like other gases, the radium-emanation is somewhat soluble in water. It disintegrates at the rate of one-half in about four days, and since the radio-active products, into which the emanation disintegrates, decay at the rate of one-half in a few minutes, it follows that the total radiation from the emanation and the subsequent disintegration-products, decreases at the same rate as the emanation, namely one-half in about four days.

When water with emanation in solution, is left in an open bottle, the emanation diffuses out, and, if the water is shaken up or otherwise disturbed, the process of diffusion of the emanation is accelerated. From ten to thirty percent. of the emanation, in solution in water, may be lost by pouring from one vessel to another.

The strength of radio-active water, is usually expressed in Mache Units per liter. Radio-active water of 2700 Mache Units contains, per liter, as much emanation as is emitted in thirty days by one microgram of radium (1 Mache Unit equals 0.001 electro-static units, one of which equals $3.33 \times 10^{-10}$ amperes). The radio-activity of water is measured by a fontactoscope, which is an electroscope with a chamber for ionized air and a scale for measuring and timing the discharge. The instrument is standardized by first testing a solution of a knowrs amount of radium chloride which has been sealed thirty days. Great care must be used in sampling the water.

Water is charged, either by dissolving the soluble bromide or chloride of radium or by submerging the insoluble sulphate. The latter is more economical, but the sulphate must be in a minute state of subdivision and must present the largest possible surface.

There are several ways of accomplishing this:

1. Precipitating the sulphate on asbestos and placing it in a porous cell.

2. Mixing it with charcoal and forming into slabs.

3. Mixing it with cement and forming balls.

4. Mixing it with clay and firing it, forming terra cotta.

Most of these processes are protected by patents. The advantage of using 
an insoluble salt is, that it can be employed repeatedly and its use continued indefinitely. The terra cotta rods can be used eighteen hundred years and still have half their radium content available.

Moreover they avoid introducing into the organism a permanent radio-active body, as is done if a soluble salt is administered.

While a given amount of radium always emits a constant and uniform amount of emanation, the proportion given out by an insoluble salt depends upon its state of sub-division.

In the insoluble salts, most of the emanation is occluded by the salt itself; in compact form, the sulphate will only yield two and a half percent., while if it is finely powdered and divided, so that is presents a large surface, ten percent. can be obtained.

A uniform strength of emanation, is obtained when the same amount of radium sulphate, is held in the same state of sub-division, submerged in the same volume of water, for the same length of time.

If it is desired to prepare doses of one hundred Mache Units, and the sulphate can be held in such a state that ten percent. of its emanation is available (as is the case when distributed through porous terra cotta) it will be convenient to use an amount of radium which would yield two thousand Mache Units and submerge for four days in tightly closed containers, when one half of ten percent. or one hundred Mache Units will be obtained.

The stronger natural springs contain from one to two hundred Mache Units per liter, with which they are charged while flowing over radio-active minerals or passing through cavities where the emanation has collected. The reason why many mineral waters, when drunk at the springs, give therapeutic results unattainable when they are bottled and transported, is the speedy dissipation of the fugitive emanation, which is reduced to one half in four days unless there is a source for its renewal. The means of renewing the radio-activity of bottled waters or of charging any water with emanation, are afforded by the above-mentioned devices, and the physician may thus prescribe a course of treatment which can be carried out with precision in the patient's home.

The chief effect of the radiations from radium and its disintegration products, is to produce an ionization of the atoms of whatever substance the rays penetrate. Chemical effects follow as a secondary result of the ionization. Von Noorden and Falta say that, "in contradistinction to all other forms of electrotherapy, we possess in the radio-active substances a means of carrying electrical energy into the depths of the body, and there subjecting the juices, protoplasm and nuclei of the cells, to an immediate bombardment by explosions of electrical atoms. We may therefore designate this internal treatment with radio-active substances, internal electro-therapy.

\section{DISCUSSION.}

Prof. SAYRE:-What have been the therapeutical results obtained from the use of these radio-mineral waters; have they been of a very reliable character?

$D_{R}$. Schirfretin :-I do not feel competent to answer that, but whenever sufficient results have been obtained to make a demand from physicians of standing, it is well for the pharmacists to be in a position to supply it. All kinds of claims are made, and a great many cases which have been treated have been brought to my attention, but not being a physician I hesitate to express an opinion. I am willing to mention if I may, some of the extraordinary 
things not connected with medicine, that have come to my attention, in regard to the effects of this weakly charged radium water, upon plants, for example. They have tried the experiment of taking two plants of exactly the same kind, age, and size, with the same number of leaves, etc., and watering them with the same amount of water, and in the same kind of soil, etc., the only difference being that the water applied to one plant was charged with radium emanations, and at the end of ten days, the plant so treated was four times as big as the other one. There have been a great many experiments along this line, which have given some very interesting results. If you use a thousand Mache Units, you will wither the plant; if you give a man 10,000 Mache Units you do not seem to hurt him.

DR. RusBy :-It may be interesting to you to know, that ever since last October I have been engaged in a systematic study of the effects of radium on crop production. I have charge of the observation of a farm of 130 acres at Northfield, near Cleveland. There we have five plots of each crop, and these plots receive different quantities of radium, one of the plots receiving no radium whatever. At the same time I am in direct charge of a small plot of an acre and a half at Nutley, New Jersey, near my home, and there $I$ have had all these crops for experimentation. The results have been of the greatest interest and of the greatest importance. In the case of radishes I have secured an increase of 70 per cent. in the weight of the crops. One of the most interesting things was the result obtained with radishes grown under glass in the winter time, during February, when the amount of sunliglit was very slight, because it was stormy during the whole month and there was snow on the glass during a large part of the time, excluding the sunlight. While the roots, the radishes themselves, increased 20 per cent. in weight, the tops, or the green parts, decreased 17 per cent., showing that the plant produced more food with less action of sunlight on leaf tissues.

A European investigator has shown that the amount of the starch in the plant in the morning was greater than it was the night before, under the influence of radium; the supposition has heretofore been that starch can form only in sunlight and is consumed in darkness. The experiment shows that radium causes stanch production during the night, when the plant can not receive the rays of the sun.

Furthermore, in the same soil from which a crop has been taken, if a new crop is planted, without any additional amount of radium, the results are greater even than they were before, instead of being less.

As Dr. Schieffelin says, an excessive amount of radium emanation is injurious. Take, for instance, the amount of radium which produced an increase of 22 per cent. If that amount be increased four-fold, the crop is stunted, and will not grow at all; the plant will grow up two or three inches and has the appearance of having been poisoned, and: a crop cannot be produced.

I have made an arrangement with the New York Times to publish in their paper about the first of October, an illustrated account of these operations, and if any of you want to watch for that, you can get it.

The experiments show that radium not only results in the practical improvement of agriculture, but that it acts on the protoplasm of animals in the same way that it does upon the protoplasm of plants.

I also want to say if any of you will write to the Standard Chemical Company of Pittsburgh they will send you a little pamphlet on terms used in the measurement of radium. It is a very good one and it shows how through a misunderstanding of terms, a lot of errors have crept in.

Dr. Scriefrelin :-I want to speak of one other thing, in the hope that somebody else will testify, Prof. Rusby himself, perhaps, and that is reported by the man who invented these terra cotta rods. They are just as good for charging the air with radium emanation as for charging water with it. One experiment was made of taking a couple hundred eggs, which were all supposed to be fertile, and putting one hundred in one incubator and one hundred in another; the air in one incubator was kept charged with radium emanation during the period of incubation. Otherwise both incubators were kept under ordinary conditions. Eighty of the eggs hatched in the radium-charged incubator, while only thirty-two of the eggs hatched in the other, and the chickens hatched in the incubator which was not charged with radium, were a half ounce lighter in weight than the chickens hatched in the other.

Mr. RUSBY:- It is certainly true that the air can be charged with emanation, because it is on that principle that the electroscope, for measuring radium emanation, is based. It is because the air in the measuring instrument, is charged with radium emanation, that it becomes capable of conducting electricity and by such conduction the electroscope works.

DR. Schiefrein :- What the pharmacist should prepare for is to meet the demand on the part of the physicians. Since I have been interested in this subject a number of physicians have used these different emanators, and the demand appears greater at the present moment than the different companies are able to supply; as least, that is what I am informed. Now, that is going to be the real test. I have always said that anything that has to be sold, as a remedy, by advertising, does not belong in the Materia Medica, but the moment that physicians continue to repeat orders for a preparation, without it being constantly advertised, 
it begins to look as though it had a permanent place in the Materia Medica. I think it is a little too soon to say anything definite about radio-active water, except that for centuries people have been taking treatment at Wildbad-Gastein springs, which contain radium emanation, with beneficial results, which ought to be an eloquent argument in favor of the treatment, with radium emanations, of gout, rheumatism, etc.

Baron Liebig, over 70 vears ago, went to Gastein and took the treatment of the waters and was cured, and he said, "I am going to find out what is in that water that cured me," and he analyzed it, and took great pains in investigating it, but when he had finished, he said there was nothing in the water that chemistry at that time could detect, but that there must be something, some influence, some magnetic, or electrical influence, which causes this wonderful power in the water. And he was right, because this power is not chemical, it is electric. These little alpha particles have two positive charges of electricity to each helium atom, and they are volleyed out like volleys from a battleship, constantly at the rate of 136 millions per second from a milligram of radium. We cannot get around that; that has been shown, and if you get some of them inside of you, it is not surprising if they produce some effect.

MR. JoSEPH WEINSTEIN, of New York:-How do the European mineral waters compare with the domestic mineral waters, as to being charged with emanation, say Saratoga Springs?

Dr. Schiefredin :- They have a little, but have not been found to be very strongly radioactive.

Mr. WeInstein :-So that they do not compare at all with the European springs?

DR. Schiefrein :-A lot of them are very weak. I think Saratoga Springs are only faintly radio-active. The Hot Springs of Arkansas are radio-active. All springs are more or less radio-active, but most of them are very weak.

Mr. Weinstern :-Are the Hot Springs, of Virginia, radio-active?

Dr. Schteffelin :-Yes.

Dr. WolfF:-The Bureau of Standards will soon be ready to provide a standard radium content. That matter is receiving attention now, and we have also been considering radium preparations, and the Bureau has some of the official copies of the radium standards which have gained international recognition.

DR. Schiefredin:-Will we be able to get these?

DR. WOLFF:-I think so, shortly.

Dr. Schiefrelin:-I am glad to hear that. These emanators have been loaned by the manufacturers in the porous earthenware cups (indicating) and within that are the holders that contain the sulphate of radium. I am sorry to have to take this away, but I have another appointment in the evening and I will soon have to leave.

Mr. JosEPH FEIL, of Cleveland:-I would like to get a description of that arrangement again. You say the radium is kept in a tube, which has an opening at the end, or how is that arranged?

DR. Schrefremin:-There is another tube beyond the orifice, with vacuum too. The other tube is a brass tube, and that is where the electrical connection occurs; you know if you connect two conductors with an electrical battery and bring them together a spark will jump across. That is the principle upon which that works. The little helium atom, charged with two charges of electricity closes the circuit and makes a very delicate electrometer give a little jerk.

Mr. Jовм K. Trum, of Philadelphia:-Dr. Schieffelin says there are some things which he would rather not talk about, which I think should be talked about. His remarks about the statement made by the physician who claims that the taking of the soluble radium salt may produce cancer, as he said the experiments have shown, show that there is a great possibility of this being true, and if so, it would be a great danger to the public.

There seems to be a strange silence on the part of the leaders of surgical science as to the value of radium in the treatment of cancer. And from private conversations with physicians one is led to believe that in some cases where radium has been applied to cancer patients, there seemed to have been at first an improvement, but that subsequently the patients died, three, six months, or a year later, with a diffused carcinomatous condition. So therefore there is a possibility that the application of radium may be dangerous, not only in applying it to the carcinoma, but by drinking it in water. If these radjum waters are to become matters of commerce, there should be some regulation or control. It seems to me that radium ought to be more thoroughly understood, and there should be something done to see that the emanations alone are in the water, and not soluble salts. The public should be protected at all costs.

A MEMBER:-The theory that radium might cause cancer has been experimentally disproved. There is no longer any ground for devoting any attention to that. The British Medical Association at its meeting decided that it was time the medical profession and the public should know that in cases of superficial cancer, where the cancers are in places where they can be reached, the only appropriate treatment is the radium treatment. Furthermore 
the British Medical Association decided that the use of surgery in fighting cancer was a thing of the past. That is the last announcement which has been made.

DR. Schiefremin :-I will pass around some of these things for your inspection. These are the earthenware rods, and this is a sample of pitchblende, an extremely handsome sample. You can determine it from its looks, and also from its weight. Uranium is one of the heaviest of all the elements, and for millions of years this uranium has been breaking down to form radium, and this radium is breaking down to form a new element. It is something that we never dreamed of. It is really a transmutation.

This little terra cotta rod does not contain the radium as a little lump, but the radium is distributed all the way through it. Before he rod is made the radium is dispersed through a powder and mixed with the clay, and then the rod is baked.

I wanted to speak about the best authors on radio-activity at the present time. These books (indicating) are very recent. This book is by Paul Lazarus, and is a collection of the best papers on the therapeutic effect of radium.

This book is Soddy's "Interpretation of Radium," which was printed a year ago; and of course, the classic book is Madam Curie's work in two large volumes, which I do not have here.

The last book of ail is "Radio-active Substances and their Radiations" by Sir Ernest Rutherford, who did much of his work at McGill University, Canada. This book has gone through a number of editions, and this is the 1913 Edition, and every page is full of important data and reasoning, and there are 664 nages in the book. It is only since 1901 , that they have been doing much on this subject. These gases and elements are very recent ones, and yet we know as much, if not more, about their properties, as we do of some of the elements that have been known for a hundred years.

I neglected to call your attention to these tables (indicating charts on wall). The Curie is the regular standard, and that is the amount of emanation that is given off by 2 gram of radium. Now, this table shows how very small a Mache unit is because it takes 2700 mache units to make a microcurie.

\begin{tabular}{lccc}
\multicolumn{4}{c}{ (I abls above referred to.) } \\
Curie & Milicurie & Microcurie & Mache Units \\
1 & 1000 & 1000000 & 2700 millions \\
0.001 & 1 & 1000 & 2700000 \\
0.000001 & 0.001 & 1 & 2700 \\
0.00000000037 & 0.00000037 & 0.00037 & 1
\end{tabular}

DR. Schrefreain :-I would also like to call your attention to this table, showing the strength of various springs in Europe:

Radio-active Strength of Natural Spring Waters Measured in Mache Units Per Liter.

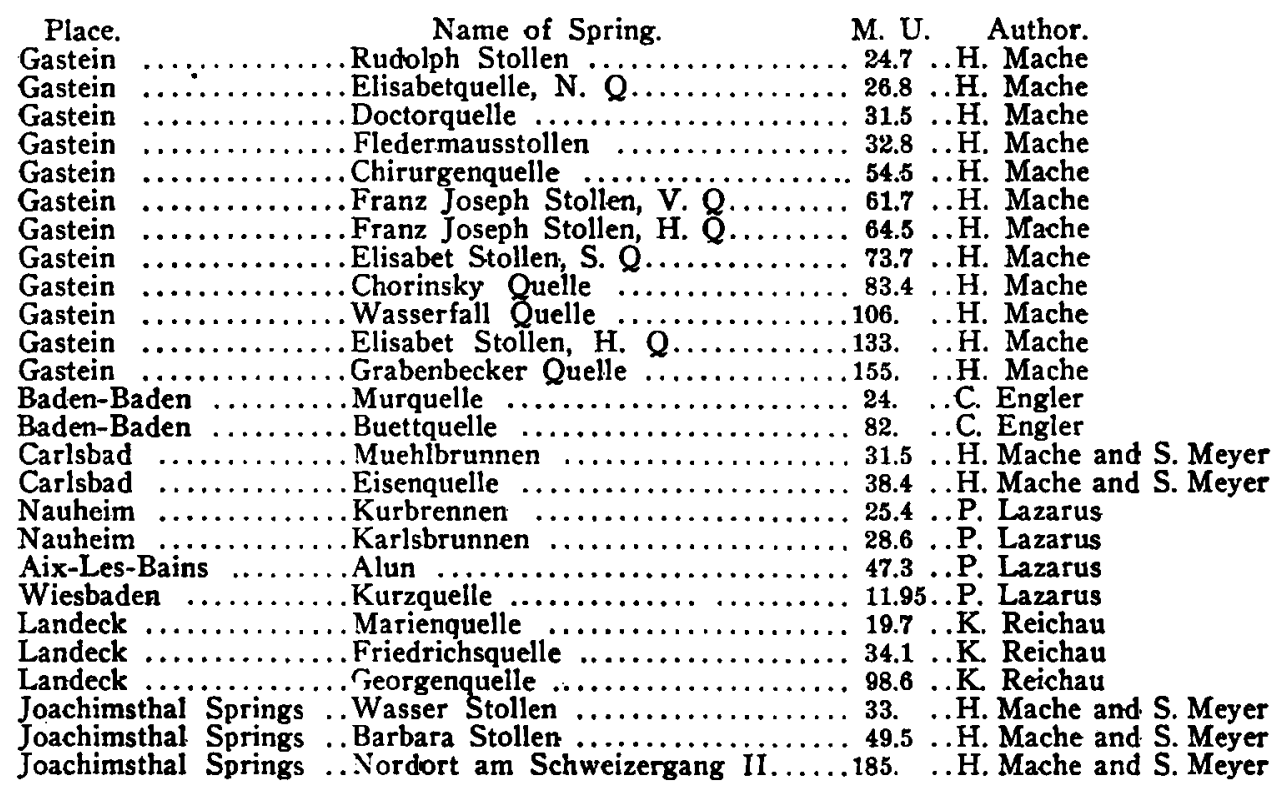

